\title{
Contents, Vol. 15, 1906
}

\section{Inhalts -Verzeichnis.}

Opig -inalarbeiten.

Asmus, E., Zur traumatischen Spätablösung der Netzhaut 444 Bach, L., Die pathologische

Anatomie der reflektorischen

Pupillenstarre 487

Blaskovics, L. v., Ueber Bindehaut- und Tarsusexzisionen

bei Trachom 391

Collin, R., Zur Methodik klinischer Farbensinn-Unter-

suchungen 305

Enslin, Eduard, Ueber Blausehen nach Starausziehung . 136

- $\quad$ - Keratitis parenchymatosa und Trauma .... 227Feilchenfeld, W., Erwerbsfähigkeit bei

Augenschäden . 126Fick, A. E., Ueber Durchschneidung der Ciliarnerven mit

Schonung des Sehnerven

Fuchs, Ernst, Ueber Komplikationen der Heterochromie 191

Gallus, Edwin, Hyperopie und Diabetes mellitus . . 319Gutmann, Adolf, Beitrag zu den

Erkrankungen des Auges

in ihrenBeziehungen zudenNasennebenhöhlenempyemen $\quad$ 403Köllner, H., Erfahrungen an 1284

Kataraktextraktionen mit

Iridektomie $\quad 506$

Koerber, H., Iriszeichnung und Irisgefässe. (llierzu Taf. Ill) 110

Koster-Gzn, W., Kalium chloricum in der Augenheilkunde 524Kowalewski, R., Ueber metastatischen Aderhautkrebs.

(Hierzu Taf. II) 21

Krause, Richard, Ueber Chrysarobineinwirkungauf das Auge 233Kuhnt, Hermann, Ueber

Lidspaltenerweiterung mit Be-

nutzung von kutanem Gewebe

- $\quad$ - Zur Behandluog frischer, komplizierter, penetrierender

Yerletzungen der Hornhaut 312

Laspeyres,Kurt, Angiolipom des Augenlides und der Orbita.

(Hierzu Taf. IV) 527

Meller, J., Ueber die Beteiligung der Orbita und des Auges

an den lymphomatösen Frozessen 538

Michel, v., Ueber das Yorkommen von Amyloid am Aug-

apfel und an den Augengefässen. (Hierzu Taf. 1) . 13 Pause, G,, Ueber Dauererfolge der opeïativen Behandlung

der hochgradigen Kurzsichtigkeit 115, 212

- $\quad$ - Beitrag zur Lehre von der Kurzsichtigkeit .... 435Reis, Wilh., Zur Aetiologie und

Genese der Lochbildung in 
der Macula lutea [Retinitis atrophicans centralis(Kuhnt)] 37Ruhwandl, Franz, Ausgedehnte Reste der fötalen Augen-

gefässe 245

Sachs, M., Spontane Pulsation einer atypischen, nahe der

Macula gelegenen Wirbelvene . 537

Schirmer, Otto, Ueber Keratitis ex acne rosacea . . 501Scholtz,Kornél, Die geographische

Yerbreitung des Trachoms

in Ungarn 105

Spengler, Erich, E⿺辶⿸ $1 / 8$ Gefahr des Argyrols 441

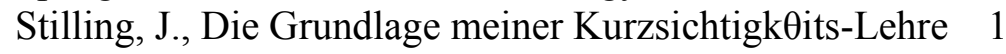

$-\mathrm{IV}-$

Verderame, Ph., Klinische und experimentelle Beiträge zur

Frage der subkonjunktivalen lnjektionen . . . 289, 411

Vogt, Alfred, Experimentelle Untersuchungen über die Be-deutung der chemischen

Eigenschaften der basischenAnilinfarbstoffe fur deren schädliche Wirkung auf

dieAugenschleinabaut 58

We ill, Georges, Kontasion des Auges mit nachträglicher

Netzhautablösung $\quad 140$

Bepieht übep die deutsche ophthalmologisehe Litepatup.

Diagnostik und Extraktion von Fremdkörpern (1905). Yon

Dr. Eduard Asmus in Düsseldorf 546

Mikroorganismen (I. und II. Semester 1904). Von Priv.-Doz.

Dr. Helbron in Berlin 155

PathologischeAnatomie (1905). Von Prof. v.Mich el inBerlin 247,336 Spezielle Patliologie und Therapie (I. und II. Semester 1904).

Von Dr. Schrader in Gera . .143, 270, 323

Anatomie des Auges (I. Semester 1905). Von Prof. Dr.

Sobotta in Würzburg .459

Physiologie des Gesichtssinnes (II. Semester 1904). Von

Priv.-Doz. Dr. W· Trendelenburg in Freiburg i. B. 445

Bepieht übep die ausländisehe ophthalmologisehe Litepatup.

Bericht über die amerikanisclie Literatur (II. Semester 1904).

Von R. Denig in New-York 357

Bericht über die holländische ophthalmologisehe Literatur (II. Semester 1904). Von Dr. G. J.

Schoute in Amsterdam und Prof. Dr. W. Koster-Gzn. in Leiden 72

Bericht über die holländische ophthalmologisehe Literatur (I. Semester 1905). Von Dr. G. J.

Schoute iu Amsterdam und Prof. Dr. W. Koster-Gzn. in Leiden 361

Bericht über die skandinavische ophthalmologisehe Literatur(1. Semester 1905). Von Doz. Dr.

E. Forsmark inStockholm 84

Bericht über die ungarische ophthalmologisehe Literatur(I. und II. Semester 1905). Von Prof. Dr.

EmilGrósz in Budapest $\quad 560$

Gesellsehaftsbepiehte.

Berliner ophthalmologisehe Gesellschaft .... 93, 181, 369 Ophthalmologisehe Gesellschaft in

Wien . . 86, 375, 476, 572

Chicago Ophthalmological Society 567

Colorado Ophthalmological Society 568 
XVII. Versammlung der italienischen ophthalmologischen Gesellschaft 162

Société d'ophtalmologie de Paris $178,372,568$

Offene Koppespondenz $\quad 100,186,387,482,576$

Thepapeutisehe Umsehau ... 95, 182, 283, 381, 479, 574

Unfall- und Vepsichepung skunde 367, 472 